\title{
molecules
}

ISSN 1420-3049

www.mdpi.com/journal/molecules

Article

\section{Synthesis and Antimicrobial Activity of Some New Thieno[2,3-b]thiophene Derivatives}

\author{
Yahia Nasser Mabkhot ${ }^{1, *}$, Nabila Abdelshafy Kheder ${ }^{2, \dagger}$ and Ahmad M. Farag ${ }^{2}$ \\ 1 Department of Chemistry, Faculty of Science, King Saud University, P.O. Box 2455, \\ Riyadh 11451, Saudi Arabia \\ 2 \\ Department of Chemistry, Faculty of Science, Cairo University, Giza 12613, Egypt \\ $\dagger$ Current address: Department of Pharmaceutical Chemistry, Faculty of Pharmacy, \\ King Khalid University, Abha 61441, Saudi Arabia \\ * Author to whom correspondence should be addressed; E-Mail: yahia@ksu.edu.sa; \\ Tel.: +966-1-467-5898; Fax: +966-1-467-5992.
}

Received: 28 March 2013; in revised form: 10 April 2013 / Accepted: 16 April 2013 / Published: 19 April 2013

\begin{abstract}
A series of thieno[2,3-b]thiophene moiety-containing bis-cyanopyrazoles 5a-c, bis-aminopyrazole 9 and bis-pyridazine derivatives $\mathbf{1 1}$ were synthesized and evaluated in vitro for their antimicrobial potential. The antimicrobial activity of some selected products was evaluated and showed good results. 5,5'-(3,4-Dimethylthieno[2,3- $b]$ thiophene-2,5diyl)bis(3-acetyl-1-(4-chlorophenyl)-1H-pyrazole-4-carbonitrile) (5d) was found to be more potent than the standard drug amphotericin B against Geotricum candidum and equipotent to amphotericin B against Syncephalastrum racemosum. In addition, it was found to be equipotent to the standard drug Penicillin G against Staphylococcus aureus. Moreover, it was more potent than the standard drug streptomycin against Pseudomonas aeruginosa and Escherichia coli.
\end{abstract}

Keywords: thieno[2,3-b]thiophene; nucleophilic addition; hydrazonoyl halides; bis-pyrazole; antimicrobial activity 


\section{Introduction}

Thieno[2,3-b]thiophenes possess important biological activities, including antiinflammatory [1,2], antimicrobial [3], analgesic [4] properties, antiproliferative activity [5], antagonism of $\alpha 1$ adrenoceptors [6] and prevention of cartilage destruction in articular diseases [7], In addition, thieno[2,3-b]thiophenes have shown useful reactivity as co-polymerization agents [8] and as semiconductors [9]. On the other hand, the synthesis of pyrazoles remains of great interest owing to their wide applications in the agrochemical and pharmaceutical industry due to their herbicidal, fungicidal, insecticidal, analgesic, antipyretic and anti-inflammatory properties [10,11]. Pyridazine compounds are also commonly used as anticancer [12], antituberculosis [13], antihypertensive [14], antifungal [15,16], or antimicrobial [17-20] agents, due to their intense biological activity. Encouraged by all these findings and in continuation of our ongoing research program investigating the utilisation of 3,3'-(3,4-dimethylthieno[2,3-b]thiophene-2,5-diyl)bis(3-oxopropanenitrile) (1) as a versatile and useful building block for the synthesis of a wide variety of bis-heterocyclic systems [21-25], we report in the present work an efficient and rapid method for the synthesis of a series of bis-cyanopyrazole, bis-aminopyrazole and bis-pyridazine derivatives containing thieno[2,3-b]thiophene as a base unit.

\section{Results and Discussion}

\subsection{Chemistry}

Treatment of 3,3'-(3,4-dimethylthieno[2,3-b]thiophene-2,5-diyl)bis(3-oxopropanenitrile) (1) [21] with hydrazonoyl chloride 2a [26] in ethanolic sodium ethoxide solution at room temperature furnished a single product, for which the two possible structures 5a and 6a can be envisaged (Scheme 1), but elemental analyses and spectral data were in complete accordance with the bis-cyanopyrazole structure 5a. The IR spectrum of compound 5 a revealed the absence of amino and carbonyl bands. Its ${ }^{1} \mathrm{H}-\mathrm{NMR}$ spectrum revealed a singlet signal at $\delta 2.3$ corresponding to methyl protons, in addition to aromatic multiplet protons in the $\delta 7.46-8.0$ region. Moreover, the mass spectrum of product 5a exhibited a molecular ion peak at $\mathrm{m} / \mathrm{z}$ 654. Prompted by the foregoing results and to generalize this finding we also studied the reaction of the thieno[2,3-b]thiophene $\mathbf{1}$ with other hydrazonoyl chlorides 2b [27] or 2c,d [28] or 2e-g [29] under the same experimental conditions and thus obtained the respective bis-cyanopyrazole derivatives $\mathbf{5 b}, \mathbf{c}$. The structure of the isolated products $\mathbf{5 b}-\mathbf{g}$ were established from their elemental analyses and spectral data (see Experimental).

Treatment of the thieno[2,3-b]thiophene 1 with phenyl isothiocyanate, in dimethylformamide, and in the presence of potassium hydroxide, at room temperature, followed by treatment with dilute hydrochloric acid, afforded a yellow-colored product identified 2-cyano-3-mercapto-3(phenylamino)acryloyl)-3,4-dimethylthieno[2,3-b]thiophene-2-carbonyl)-3-mercapto-3-(phenylamino) acrylonitrile (8) (Scheme 2).

The structure of the latter product was confirmed on the basis of its elemental analysis and spectral data. The IR spectrum of compound 8 showed absorption bands at 3411,2207 and $1714 \mathrm{~cm}^{-1}$ corresponding to $\mathrm{NH}$, nitrile and carbonyl groups, respectively. Its ${ }^{1} \mathrm{H}-\mathrm{NMR}$ spectrum revealed a singlet signal at $\delta 2.49$ and two $\mathrm{D}_{2} \mathrm{O}$-exchangeable signals at $\delta 7.07$ and 14.07 due to methyl, $\mathrm{NH}$, and 
SH protons, respectively, in addition to aromatic multiplet protons in the $\delta 7.29-8.02$ region. Refluxing of the thieno[2,3-b]thiophene $\mathbf{8}$ with hydrazine hydrate afforded bis-aminopyrazole 9.

Scheme 1. Synthesis of bis-cyanopyrazole derivatives $\mathbf{5 a - g}$.

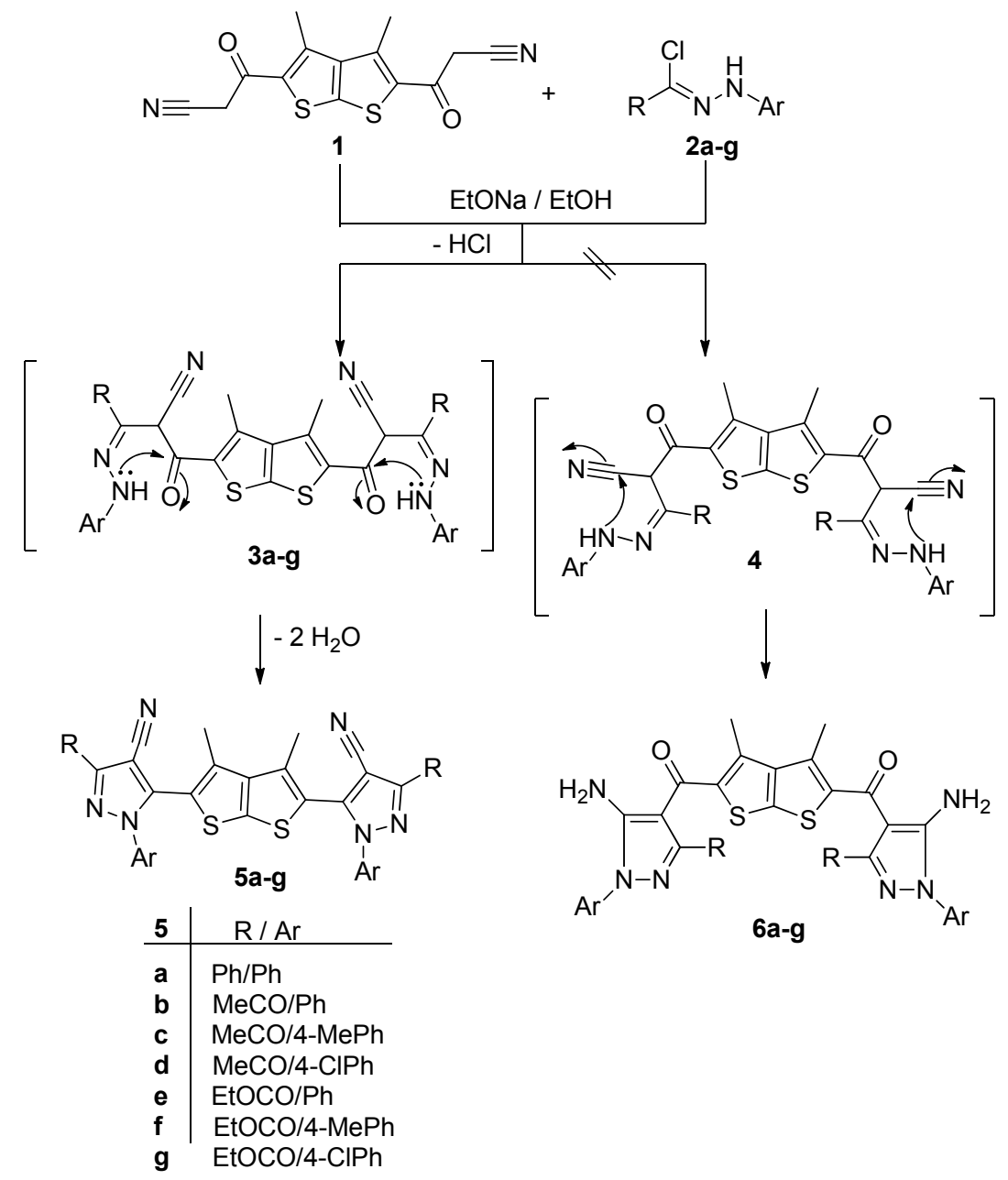

Scheme 2. Synthesis of thieno[2,3-b]thiophene 8 and bis-aminopyrazole 9.

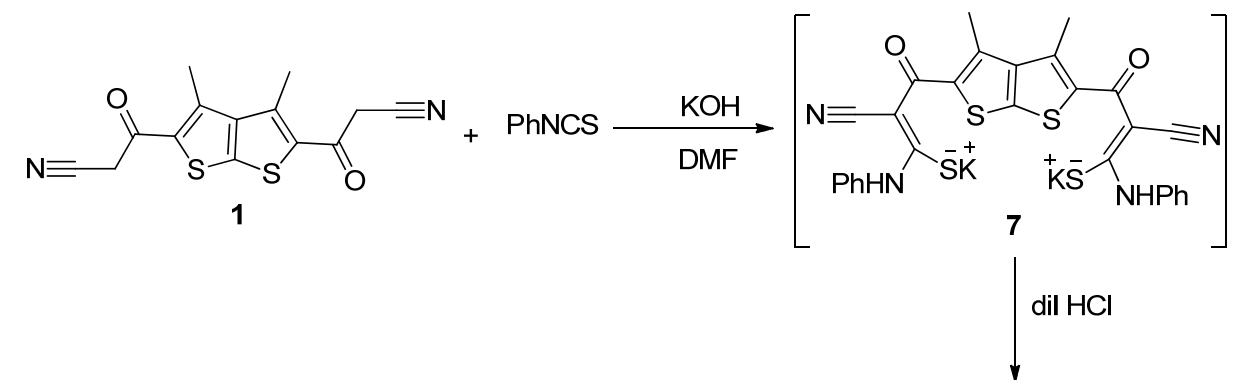
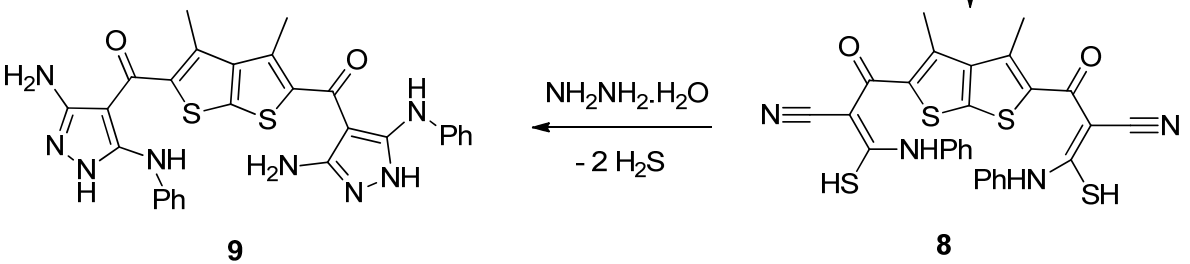
The ${ }^{1} \mathrm{H}-\mathrm{NMR}$ spectrum of 9 revealed the absence of $\mathrm{SH}$ proton and showed a singlet signal at $\delta$ 2.49 and two $\mathrm{D}_{2} \mathrm{O}$-exchangeable signals at $\delta 4.55$ and at $\delta 7.93$ corresponding to methyl, $\mathrm{NH}_{2}$, and $\mathrm{NH}$ proton, respectively, in addition to aromatic multiplet protons in the region $\delta 7.31-7.45$.

Treatment of hydrazone 10 [21] with malononitrile in DMF, afforded bis-pyridazine 11 (Scheme 3). The structure of the latter product was established on the basis of its elemental analysis and spectral data. For example, its IR spectrum revealed two absorption band at $\mathrm{v}=1651,2214 \mathrm{~cm}^{-1}$, assignable to carbonyl and nitrile groups, in addition to bands at $\mathrm{v}=3175-3410 \mathrm{~cm}^{-1}$ due to $\mathrm{NH}_{2}$ and $\mathrm{NH}$. Its ${ }^{1} \mathrm{H}-\mathrm{NMR}$ spectrum showed two singlet signals at $\delta 2.49 \mathrm{ppm}$ due to methyl groups, and two signals $\left(\mathrm{D}_{2} \mathrm{O}\right.$-exchangeable) at $\delta 4.1$ and $7.48 \mathrm{ppm}$ assignable to $\mathrm{NH}_{2}$, and $\mathrm{NH}$ protons, respectively. Moreover, the mass spectrum of the product 11 exhibited a molecular ion peak at $\mathrm{m} / \mathrm{z} 711$.

Scheme 3. Synthesis of bis-pyridazine 11.

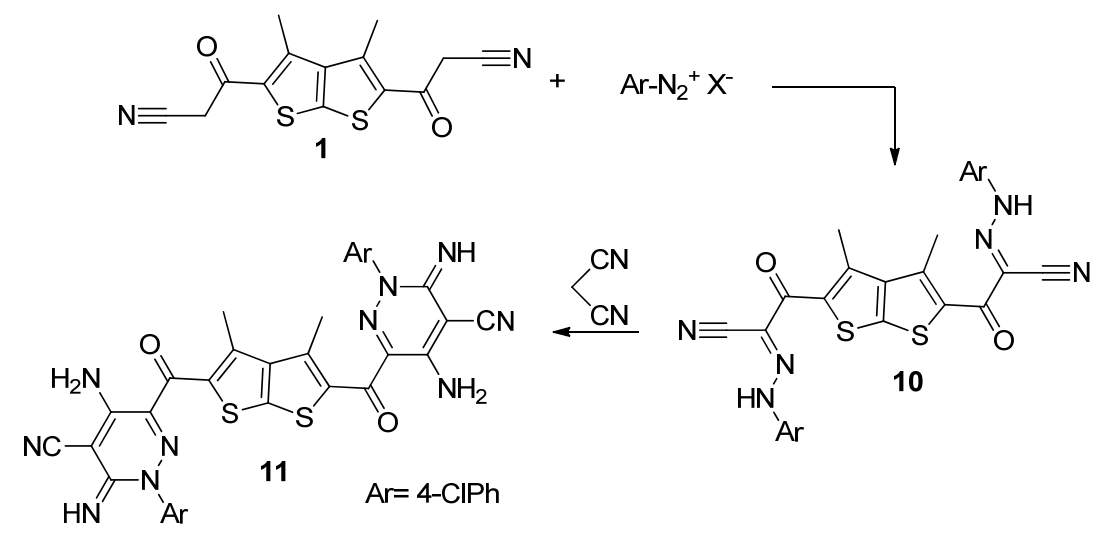

\subsection{Antimicrobial Evaluation}

The newly synthesized target compounds $\mathbf{5 b}, \mathbf{c}$ and $\mathbf{1 1}$ were evaluated for their in vitro antibacterial activity against Streptococcus pneumoniae and Bacillis subtilis as examples of Gram-positive bacteria and Pseudomonas aeruginosa and Escherichia coli as examples of Gram-negative bacteria. They were also evaluated for their in vitro antifungal potential against the fungal strains Aspergillus fumigatus, Syncephalastrum racemosum, Geotricum candidum and Candida albicans. The organisms were tested against the activity of solutions of concentrations $(5 \mu \mathrm{g} / \mathrm{mL})$ and using inhibition zone diameter (IZD) in $\mathrm{mm}$ as criterion for the antimicrobial activity (agar diffusion method). The fungicide amphotericin $\mathrm{B}$ and the bactericides penicillin $\mathrm{G}$ and streptomycin were used as references to evaluate the potency of the tested compounds under the same conditions. The results are summarized in Tables/Figures 1 and 2.

Table 1. Antifungal activities of the synthesized compounds $\mathbf{5 c , d}$ and $\mathbf{1 1}$.

\begin{tabular}{ccccc}
\hline \multirow{2}{*}{ Compound Tested } & \multicolumn{4}{c}{ Micoorganisms } \\
\cline { 2 - 5 } & $\begin{array}{c}\text { Aspergillus } \\
\text { Fumigatus }\end{array}$ & $\begin{array}{c}\text { Geotrichum } \\
\text { Candidum }\end{array}$ & $\begin{array}{c}\text { Candida } \\
\text { albicans }\end{array}$ & $\begin{array}{c}\text { Syncephalastrum } \\
\text { racemosum }\end{array}$ \\
\hline $\mathbf{5 c}$ & $17.3 \pm 0.4$ & $21.3 \pm 0.4$ & NA & $14.8 \pm 0$ \\
$\mathbf{5 d}$ & $22.4 \pm 0.5$ & $29.7 \pm 0.2$ & NA & $19.8 \pm 0.8$ \\
$\mathbf{1 1}$ & $18.9 \pm 0.3$ & $23.4 \pm 0.4$ & NA & $16.5 \pm 0.2$ \\
Amphotericin B & $23.7 \pm 0.1$ & $28.7 \pm 0.2$ & $25.4 \pm 0.1$ & $19.7 \pm 0.2$ \\
\hline
\end{tabular}

NA: No activity, data are expressed in the form of mean $\pm \mathrm{SD}$. 
Table 2. Antibacterial activities of the synthesized compounds $\mathbf{5 c , d}$ and $\mathbf{1 1}$.

\begin{tabular}{cccccc}
\hline Compound & \multicolumn{2}{c}{ Gram-Positive Bacteria } & & \multicolumn{2}{c}{ Gram-Negative Bacteria } \\
\cline { 2 - 3 } \cline { 5 - 6 } Tested & S. aureus & B. subtilis & & P. aeruginosa & E. coli \\
\hline $\mathbf{5 c}$ & $18.6 \pm 0.3$ & $20.9 \pm 0.5$ & & $17.4 \pm 0.2$ & $20.3 \pm 0.4$ \\
$\mathbf{5 d}$ & $23.8 \pm 0.5$ & $27.4 \pm 0.6$ & & $20.7 \pm 0.2$ & $26.8 \pm 0.2$ \\
$\mathbf{1 1}$ & $20.4 \pm 0.4$ & $23.1 \pm 0.2$ & & $19.6 \pm 0.1$ & $22.2 \pm 0.5$ \\
Penicillin $\boldsymbol{G}$ & $23.8 \pm 0.2$ & $32.4 \pm 0.3$ & & - & - \\
Streptomycin & - & - & & $20.3 \pm 0.1$ & $24.9 \pm 0.3$ \\
\hline
\end{tabular}

NA: No activity, data are expressed in the form of mean \pm SD. Mean zone of inhibition in $\mathrm{mm} \pm$ Standard deviation beyond well diameter $(6 \mathrm{~mm})$ produced on a range of environmental and clinically pathogenic microorganisms using $(5 \mathrm{mg} / \mathrm{mL})$ concentration of tested samples.

Figure 1. Antifungal activities of the synthesized compounds 5c,d and $\mathbf{1 1}$ against micoorganisms-1. Aspergillus fumgatus; 2. Geotrichum candidum; 3. Candida albicans; 4. Syncephalastrum racemosum.

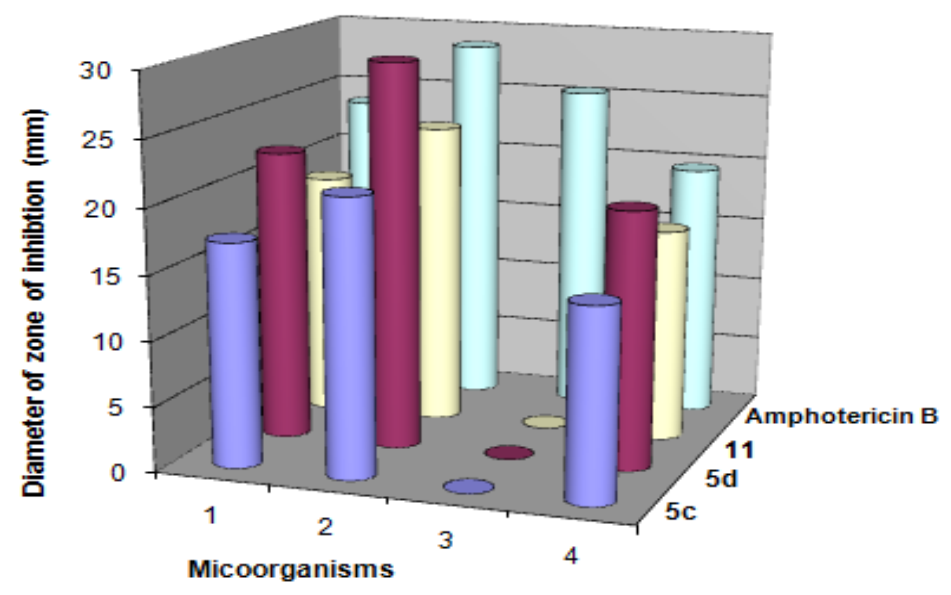

Figure 2. Antibacterial activities of the synthesized compounds $\mathbf{5 c , d}$ and $\mathbf{1 1}$ against micoorganisms-1. Staphylococcus aureus; 2. Bacillis subtilis; 3. Pseudomonas aeruginosa; 4. Escherichia coli.

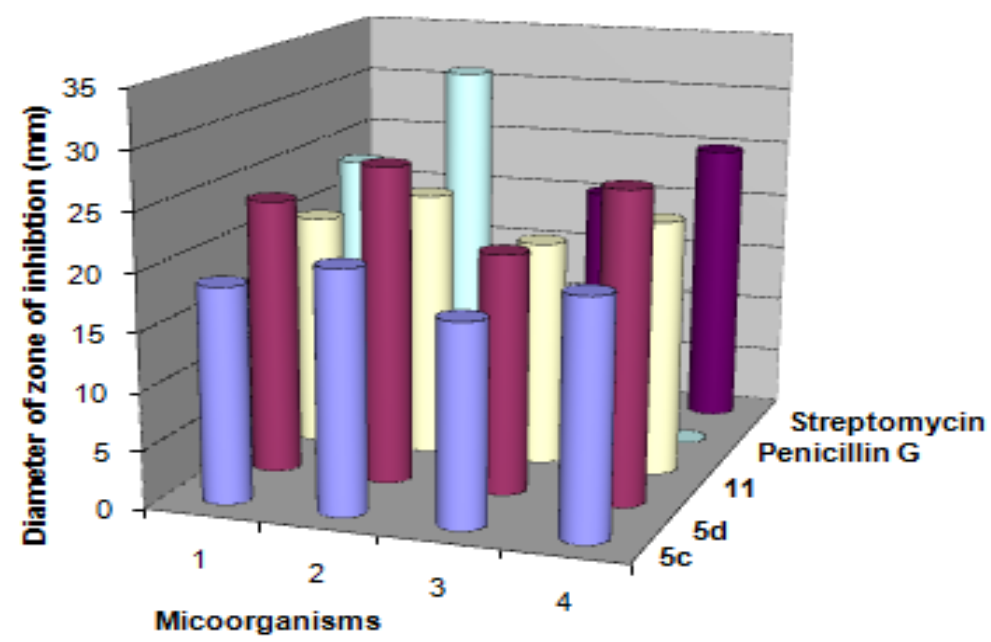


The results in Table 1 revealed that 5,5'-(3,4-dimethylthieno[2,3- $b$ ]thiophene-2,5-diyl)bis(3-acetyl1-(4-chlorophenyl)-1H-pyrazole-4-carbonitrile) (5d) was found to be more potent than the standard drug Amphotericin B against Geotricum candidum. Also, it was equipotent to the standard drug, amphotericin B against Syncephalastrum racemosum. In addition, it was found to be equipotent to the standard drug penicillin G against Staphylococcus aureus. Moreover, it was more potent than the standard drug streptomycin against Pseudomonas aeruginosa and Escherichia coli. All compounds exhibited almost no activity against Candida albicans. The structure antimicrobial activity relationship of the synthesized compounds $\mathbf{5 c}$ and $\mathbf{5 d}$ revealed that the maximum activity was attained with compound $\mathbf{5 d}$, having a pyrazole nucleus with chloro substituent in the phenyl group.

\section{Experimental}

All melting points were measured on a Gallenkamp melting point apparatus (Weiss-Gallenkamp, London, UK). The infrared spectra were recorded in potassium bromide disks on a pye Unicam SP 3300 and Shimadzu FT IR 8101 PC infrared spectrophotometers (Pye Unicam Ltd. Cambridge, England and Shimadzu, Tokyo, Japan, respectively). The NMR spectra were recorded on a Bruker VX-500 NMR spectrometer (Varian, Palo Alto, CA, USA). ${ }^{1} \mathrm{H}-\mathrm{NMR}$ spectra were run at $500 \mathrm{MHz}$ and ${ }^{13} \mathrm{C}$-NMR spectra were run at $125 \mathrm{MHz}$ in deuterated dimethylsulphoxide (DMSO- $d_{6}$ ). Chemical shifts were related to that of the solvent. Mass spectra were recorded on a Shimadzu GCMS-QP 1000 EX mass spectrometer at $70 \mathrm{e} . \mathrm{V}$. Elemental analyses were carried out at the Micro-analytical Center of Cairo University, Giza, Egypt. The biological evaluation of the products 5c,d and $\mathbf{1 1}$ were carried out in the Medical Mycology Laboratory of the Regional Center for Mycology and Biotechnology of Al-Azhar University, Cairo, Egypt. Thieno[2,3-b]thiophene 1 [21], hydrazonoyl chlorides 2a [26], 2b [27], $\mathbf{2 c}, \mathbf{d}$ [28], 2e-g [29], and hydrazone 10 [21] were prepared following the literature procedures.

\subsection{Reaction of Thieno[2,3-b]thiophene $\mathbf{1}$ with Hydrazonoyl Halides}

General Procedure

Thieno[2,3-b]thiophene $1(0.3 \mathrm{~g}, 1 \mathrm{mmol})$ was added to an ethanolic sodium ethoxide solution [prepared from sodium metal $(46 \mathrm{mg}, 2 \mathrm{mmol})$ and absolute ethanol $(20 \mathrm{~mL})$ ] with stirring. After stirring the resulting solution for $15 \mathrm{~min}$, the appropriate hydrazonoyl halide $\mathbf{2 a}-\mathbf{g}(2 \mathrm{mmol})$ was added portionwise and the reaction mixture was stirred further for $12 \mathrm{~h}$ at room temperature. The solid that formed was filtered off, washed with water and dried. Recrystallization from the proper solvent afforded the corresponding pyrazole derivatives $\mathbf{5 a}-\mathbf{g}$ in $45-55 \%$ yields.

5,5'-(3,4-Dimethylthieno[2,3-b]thiophene-2,5-diyl)bis(1,3-diphenyl-1H-pyrazole-4-carbonitrile) (5a). Yield 48\%, mp > $300{ }^{\circ} \mathrm{C}(\mathrm{DMF} / \mathrm{EtOH})$; IR $(\mathrm{KBr}) \vee \max : 2226(\mathrm{C} \equiv \mathrm{N}), 2909$ (aliphatic $\left.\mathrm{CH}\right) \mathrm{cm}^{-1}$; ${ }^{1} \mathrm{H}-\mathrm{NMR}: \delta 2.3\left(\mathrm{~s}, 6 \mathrm{H}, 2 \mathrm{CH}_{3}\right), 7.46-8.0(\mathrm{~m}, 20 \mathrm{H}, \mathrm{ArH}) ;{ }^{13} \mathrm{C}-\mathrm{NMR}: \delta 13.88,93.26,114.24,115.42$, $122.91,125.08,126.46,129.21,129.49,129.92,133.65,138.08,140.27,142.54,144.54,146.09$, 152.23. MS $m / z(\%) 657$ (11.64), 656 (30.71), $655\left(\mathrm{M}^{+}, 60.86\right), 654$ (63.43), 637 (5.22), 620 (5.82), 619 (6.42), 166 (26.18), 77 (87.63). Anal. Calcd for $\mathrm{C}_{40} \mathrm{H}_{26} \mathrm{~N}_{6} \mathrm{~S}_{2}$ (654.80): C, 73.37; H, 4.00; N, 12.83; S, 9.79. Found: C, 73.28; H, 4.10; N, 12.77; S, 9.67\%. 
5,5'-(3,4-Dimethylthieno[2,3-b]thiophene-2,5-diyl)bis(3-acetyl-1-phenyl-1H-pyrazole-4-carbonitrile) (5b). Yield 45\%, mp > $300{ }^{\circ} \mathrm{C}(\mathrm{DMF} / \mathrm{EtOH})$; IR $(\mathrm{KBr})$ v max: $1680(\mathrm{C}=\mathrm{O}), 2226(\mathrm{C} \equiv \mathrm{N}), 2915$ (aliphatic $\mathrm{CH}) \mathrm{cm}^{-1}$; ${ }^{1} \mathrm{H}-\mathrm{NMR}: \delta 2.16\left(\mathrm{~s}, 6 \mathrm{H}, 2 \mathrm{CH}_{3}\right), 2.30\left(\mathrm{~s}, 6 \mathrm{H}, 2 \mathrm{CH}_{3}\right), 7.46-7.98(\mathrm{~m}, 10 \mathrm{H}, \mathrm{ArH}) ; \mathrm{MS} m / z(\%)$ $587\left(\mathrm{M}^{+}, 12.82\right), 585$ (82.77), 464 (33.57), 166 (26.18), 77 (87.63). Anal. Calcd for $\mathrm{C}_{32} \mathrm{H}_{22} \mathrm{~N}_{6} \mathrm{O}_{2} \mathrm{~S}_{2}$ (586.69): C, 65.51; H, 3.78; N, 14.32; S, 10.93. Found: C, 65.62; H, 3.85; N, 14.44 S, 11.03\%.

5,5'-(3,4-Dimethylthieno[2,3-b]thiophene-2,5-diyl)bis(3-acetyl-1-p-tolyl-1H-pyrazole-4-carbonitrile) (5c). Yield 45\%, mp > $300{ }^{\circ} \mathrm{C}(\mathrm{DMF} / \mathrm{EtOH})$; IR $(\mathrm{KBr})$ v max: $1680(\mathrm{C}=\mathrm{O}), 2226(\mathrm{C} \equiv \mathrm{N}), 2915$ (aliphatic $\mathrm{CH}) \mathrm{cm}^{-1}$; ${ }^{1} \mathrm{H}-\mathrm{NMR}: \delta 2.16\left(\mathrm{~s}, 6 \mathrm{H}, 2 \mathrm{CH}_{3}\right), 2.30\left(\mathrm{~s}, 6 \mathrm{H}, 2 \mathrm{CH}_{3}\right), 2.79\left(\mathrm{~s}, 6 \mathrm{H}, 2 \mathrm{CH}_{3}\right) ; 7.46-7.98(\mathrm{~m}, 8 \mathrm{H}$, ArH); MS m/z (\%) $615\left(\mathrm{M}^{+}, 18.25\right), 192$ (47.53) 166 (26.18). Anal. Calcd for $\mathrm{C}_{34} \mathrm{H}_{26} \mathrm{~N}_{6} \mathrm{O}_{2} \mathrm{~S}_{2}$ (614.74): C, 66.43; H, 4.26; N, 13.67; S, 10.43. Found: C, 66.52; H, 4.36; N, 13.58; S, 10.23\%.

5,5'-(3,4-Dimethylthieno[2,3-b] thiophene-2,5-diyl)bis(3-acetyl-1-(4-chlorophenyl)-1H-pyrazole-4carbonitrile) (5d). Yield 75\%, mp $>300{ }^{\circ} \mathrm{C}(\mathrm{DMF} / \mathrm{EtOH})$; IR $(\mathrm{KBr}) \vee \max : 1680(\mathrm{C}=\mathrm{O}), 2230(\mathrm{C} \equiv \mathrm{N})$, 2990 (aliphatic $\mathrm{CH}) \mathrm{cm}^{-1}$; ${ }^{1} \mathrm{H}-\mathrm{NMR}: \delta 2.16\left(\mathrm{~s}, 6 \mathrm{H}, 2 \mathrm{CH}_{3}\right), 2.30\left(\mathrm{~s}, 6 \mathrm{H}, 2 \mathrm{CH}_{3}\right), 7.46-7.98(\mathrm{~m}, 8 \mathrm{H}$, ArH); MS m/z (\%) $655\left(\mathrm{M}^{+}, 8.25\right), 192$ (47.53) 166 (26.18). Anal. Calcd for $\mathrm{C}_{32} \mathrm{H}_{20} \mathrm{Cl}_{2} \mathrm{~N}_{6} \mathrm{O}_{2} \mathrm{~S}_{2}(655.58)$ : C, 58.63; H, 3.07; N, 12.82; Cl, 10.82; S, 9.78. Found: C, 58.72; H, 3.16; N, 12.77; Cl, 10.67; S, 9.93\%.

Diethyl 5,5'-(3,4-dimethylthieno[2,3-b]thiophene-2,5-diyl)bis(4-cyano-1-phenyl-1H-pyrazole-3-carboxylate) (5e). Yield 43\%, mp > $300{ }^{\circ} \mathrm{C}(\mathrm{DMF} / \mathrm{EtOH})$; IR (KBr) v max: $1680(\mathrm{C}=\mathrm{O}), 2230(\mathrm{C} \equiv \mathrm{N}), 2980$ (aliphatic $\mathrm{CH}) \mathrm{cm}^{-1}$; ${ }^{1} \mathrm{H}-\mathrm{NMR}: \delta 1.16\left(\mathrm{t}, 6 \mathrm{H}, 2 \mathrm{CH}_{3}\right), 2.30\left(\mathrm{~s}, 6 \mathrm{H}, 2 \mathrm{CH}_{3}\right), 4.23\left(\mathrm{q}, 4 \mathrm{H}, 2 \mathrm{CH}_{2}\right)$, 7.46-7.98 (m, 10H, ArH); MS m/z (\%) $647\left(\mathrm{M}^{+}, 17.66\right), 648\left(\mathrm{M}^{+}+1,44.79\right), 192$ (47.53) 166 (26.18), 77 (87.63). Anal. Calcd for $\mathrm{C}_{34} \mathrm{H}_{26} \mathrm{~N}_{6} \mathrm{O}_{4} \mathrm{~S}_{2}$ (646.74): C, 63.14; H, 4.05; N, 12.99; S, 9.92. Found: C, $63.25 ; \mathrm{H}, 4.13 ; \mathrm{N}, 12.83 ; \mathrm{S}, 10.00 \%$.

Diethyl 5,5'-(3,4-dimethylthieno[2,3-b]thiophene-2,5-diyl)bis(4-cyano-1-p-tolyl-1H-pyrazole-3-carboxylate) (5f). Yield 77\%, mp > $300{ }^{\circ} \mathrm{C}(\mathrm{DMF} / \mathrm{EtOH})$; IR (KBr) v max: $1680(\mathrm{C}=\mathrm{O}), 2230(\mathrm{C} \equiv \mathrm{N}), 3000$ (aliphatic $\mathrm{CH}) \mathrm{cm}^{-1} ;{ }^{1} \mathrm{H}-\mathrm{NMR}: \delta 1.16\left(\mathrm{t}, 6 \mathrm{H}, 2 \mathrm{CH}_{3}\right), 2.30\left(\mathrm{~s}, 6 \mathrm{H}, 2 \mathrm{CH}_{3}\right), 2.78\left(\mathrm{~s}, 6 \mathrm{H}, 2 \mathrm{CH}_{3}\right), 4.23$ (q, 4H, 2CH$), 7.46-7.98$ (m, 8H, ArH); MS m/z (\%) 676 (33.77), $675\left(\mathrm{M}^{+}, 24.71\right), 192$ (47.53) 166 (26.18). Anal. Calcd for $\mathrm{C}_{36} \mathrm{H}_{30} \mathrm{~N}_{6} \mathrm{O}_{4} \mathrm{~S}_{2}$ (674.79): C, 64.08; H, 4.48; N, 12.45; S, 9.50. Found: C, 64.18; H, 4.37; N, 12.38; S, 9.37\%.

Diethyl 5,5'-(3,4-dimethylthieno[2,3-b] thiophene-2,5-diyl)bis(1-(4-chlorophenyl)-4-cyano-1H-pyrazole3-carboxylate) (5g). Yield 56\%, mp > $300{ }^{\circ} \mathrm{C}(\mathrm{DMF} / \mathrm{EtOH})$; IR $(\mathrm{KBr}) \mathrm{v} \max : 1680(\mathrm{C}=\mathrm{O}), 2230$ $(\mathrm{C} \equiv \mathrm{N}), 2980$ (aliphatic $\mathrm{CH}) \mathrm{cm}^{-1}$; ${ }^{1} \mathrm{H}-\mathrm{NMR}: \delta 1.16\left(\mathrm{t}, 6 \mathrm{H}, 2 \mathrm{CH}_{3}\right), 2.30\left(\mathrm{~s}, 6 \mathrm{H}, 2 \mathrm{CH}_{3}\right), 4.23(\mathrm{q}, 4 \mathrm{H}$, $\left.2 \mathrm{CH}_{2}\right), 7.46-7.98$ (m, 8H, ArH); MS m/z (\%) $715\left(\mathrm{M}^{+}, 16.95\right), 112$ (18.34), 111 (49.94). Anal. Calcd for $\mathrm{C}_{34} \mathrm{H}_{24} \mathrm{Cl}_{2} \mathrm{~N}_{6} \mathrm{O}_{4} \mathrm{~S}_{2}$ (715.63): C, 57.06; H, 3.38; Cl, 9.91; N, 11.74; S, 8.96. Found: C, 57.18; H, $3.29 ; \mathrm{Cl}, 9.91 ; \mathrm{N}, 11.83 ; \mathrm{S}, 9.85 \%$.

2-Cyano-3-mercapto-3-(phenylamino)acryloyl)-3,4-dimethylthieno[2,3-b]thiophene-2-carbonyl)-3mercapto-3-(phenylamino)acrylonitrile (8). To a stirred solution of potassium hydroxide $(0.11 \mathrm{~g}$, $2 \mathrm{mmol})$ in DMF $(20 \mathrm{~mL})$ was added thieno[2,3- $b$ ]thiophene $1(0.3 \mathrm{~g}, 1 \mathrm{mmol})$. After stirring for $30 \mathrm{~min}$, phenyl isothiocyanate $(0.27 \mathrm{~g}, 0.24 \mathrm{~mL}, 2 \mathrm{mmol})$ was added to the resulting mixture and stirring was 
continued for $6 \mathrm{~h}$, then poured onto curshed ice containing hydrochloric acid. The formed solid product was filtered off, washed with water, dried and finally recrystallized from dioxane/ EtOH mixture to afford compound 8 in $82 \%$ yield, mp $233-234^{\circ} \mathrm{C}$; IR $(\mathrm{KBr})$ v $3411(\mathrm{NH}), 2207(\mathrm{C} \equiv \mathrm{N}), 1714(\mathrm{C}=\mathrm{O}) \mathrm{cm}^{-1}$; ${ }^{1} \mathrm{H}-\mathrm{NMR}: \delta 2.49\left(\mathrm{~s}, 6 \mathrm{H}, 2 \mathrm{CH}_{3}\right), 7.07$ (s, 2H, D ${ }_{2} \mathrm{O}$-exchangeable 2NH), 7.29-8.02 (m, 10H, ArH), 14.07 (s, 1H, $\mathrm{D}_{2} \mathrm{O}$-exchangeable $\left.\mathrm{SH}\right)$. MS m/z (\%) ( $\mathrm{M}^{+}$, not detected), 353 (7.19), 203 (6.36), 166 (6.51), 92 (15.64), 77 (71.78). Anal. Calcd for $\mathrm{C}_{28} \mathrm{H}_{20} \mathrm{~N}_{4} \mathrm{O}_{2} \mathrm{~S}_{4}$ (572.74): C, 58.72; H, 3.52; N, 9.78; S, 22.39. Found: C, 58.66; H, 3.61; N, 9.88; S, 22.24\%.

Synthesis of 3,4-dimethylthieno[2,3-b] thiophene-2,5-diyl)bis((3-amino-5-(phenylamino)-1H-pyrazol-4yl)methanone) (9). A mixture of compound 8 (0.572 g, $1 \mathrm{mmol})$ and hydrazine hydrate 98\% (2 mmol) were heated on a steam bath for $1 \mathrm{~h}$, then left to cool. The reaction mixture was triturated with ethanol and the resulting solid product was filtered off and recrystalized from DMF/ethanol to give bis-pyrazole 9 in $48 \%$ yield, $\mathrm{mp}>300{ }^{\circ} \mathrm{C}$; IR $(\mathrm{KBr})$ v $3400(\mathrm{NH}), 3465(\mathrm{NH}), 1714(\mathrm{C}=\mathrm{O}) \mathrm{cm}^{-1}$; ${ }^{1} \mathrm{H}-\mathrm{NMR}: \delta 2.49\left(\mathrm{~s}, 6 \mathrm{H}, 2 \mathrm{CH}_{3}\right), 4.55\left(\mathrm{~s}, 4 \mathrm{H}, \mathrm{D}_{2} \mathrm{O}\right.$-exchangeable $\left.2 \mathrm{NH}_{2}\right), 7.26\left(\mathrm{~s}, 2 \mathrm{H}, \mathrm{D}_{2} \mathrm{O}\right.$-exchangeable 2NH), 7.93 (s, 2H, $\mathrm{D}_{2} \mathrm{O}$-exchangeable 2NH), 7.31-7.45 (m, 10H, ArH). Anal. Calcd for $\mathrm{C}_{28} \mathrm{H}_{24} \mathrm{~N}_{8} \mathrm{O}_{2} \mathrm{~S}_{2}$ (568.67): C, 59.14; H, 4.25; N, 19.70; S, 11.28. Found: C, 59.22; H, 4.34; N, 19.61; S, 11.33\%.

\subsection{Reaction of Hydrazone $\mathbf{1 0}$ with Malononitrile}

To an ethanolic solution of hydrazone $10(0.58 \mathrm{~g}, 1 \mathrm{mmol})$ and malononitrile $(0.132 \mathrm{~g}, 2 \mathrm{mmol})$ was added few drops of piperidine and the reaction mixture was refluxed for $4 \mathrm{~h}$. The solid product was collected by filtration, washed with ethanol and purified by crystallisation from DMF to afford the bis-pyridazine 11 in 66\% yield, mp 208-209 ${ }^{\circ} \mathrm{C}$; IR (KBr) v 3175, $3244\left(\mathrm{NH}_{2}\right), 3333$ (NH) 2214 $(\mathrm{C} \equiv \mathrm{N}), 1651(\mathrm{C}=\mathrm{O}) \mathrm{cm}^{-1} ;{ }^{1} \mathrm{H}-\mathrm{NMR}\left(\mathrm{DMSO}-d_{6}\right): \delta 2.49\left(\mathrm{~s}, 6 \mathrm{H}, 2 \mathrm{CH}_{3}\right), 4.1\left(\mathrm{~s}, 4 \mathrm{H}, \mathrm{D}_{2} \mathrm{O}-\right.$-exchangeable 2NH $\left.\mathrm{NH}_{2}\right), 7.31-7.48(\mathrm{~m}, 10 \mathrm{H}, \mathrm{ArH}+2 \mathrm{NH}) ; \mathrm{MS} \mathrm{m} / z(\%) 711\left(\mathrm{M}^{+}, 30.38\right), 710$ (38.26), 272 (34.65), 244 (21.45), 111 (20.70). Anal. Calcd for $\mathrm{C}_{32} \mathrm{H}_{20} \mathrm{Cl}_{2} \mathrm{~N}_{10} \mathrm{O}_{2} \mathrm{~S}_{2}$ (711.60): C, 54.01; H, 2.83; N, 19.68; Cl, 9.96; S, 9.01. Found: C, 54.15; H, 2.78; N, 19.59; Cl, 9.95; S, 8.98\%.

\section{Conclusions}

A novel series of bis-cyanopyrazole, bis-aminopyrazole and bis-pyridazine derivatives with thieno[2,3-b]thiophene moieties were synthesized and evaluated for their antimicrobial activities. In general, most of the tested compounds was found to be equipotent or more potent than the standard drugs.

\section{Acknowledgments}

The authors extend their appreciation to the Deanship of Scientific Research at King Saud University for funding this work through the research group project No. RGP-VPP-007.

\section{References}

1. El-Kerdawy, M.M.; Yousif, M.Y.; El-Emam, A.A.; Moustafa, M.A.; El-Sherbeny, M.A. Synthesis and antiinflammatory activity of certain thienopyrimidine derivatives. Boll. Chim. Farm. 1996, 135, 301-305. 
2. Modica, M.; Santagati, M.; Santagati, A.; Cutuli, V.; Mangano, N.; Caruso, A. Synthesis of new $[1,3,4]$ thiadiazolo[3,2-a]thieno[2,3- $d]$ pyrimidinone derivatives with antiinflammatory activity. Pharmazie 2000, 55, 500-502.

3. Chambhare, R.V.; Khadse, B.G.; Bobde, A.S.; Bahekar, R.H. Synthesis and preliminary evaluation of some $N$-[5-(2-furanyl)-2-methyl-4-oxo-4H-thieno[2,3- $d$ ]pyrimidin-3-yl]-carboxamide and 3-substituted-5-(2-furanyl)-2-methyl-3H-thieno[2,3- $d$ ]pyrimidin-4-ones asantimicrobial agents. Eur. J. Med. Chem. 2003, 38, 89-100.

4. Santagati, N.A.; Caruso, A.; Cutuli, V.M.; Caccamo, F. Synthsis and phamacological evaluation of thieno[2,3- $d$ ]pyrimidin-2,4-dione and 5H-pyrimido[5,4-b]indol-2,4-dione derivatives. Farmaco 1995, 50, 689-695.

5. Egbertson, M.S.; Cook, J.J.; Bednar, B.; Prugh, J.D.; Bednar, R.A.; Gaul, S.L.; Gould, R.J.; Hartman, G.D.; Homnick, C.F.; Holahan, M.A.; et al. Non-peptide GPIIb/IIIa inhibitors. 20. Centrally constrained thienothiophene alpha-sulfonamides are potent, long acting in vivo inhibitors of platelet aggregation. J. Med. Chem. 1999, 42, 2409-2421.

6. Meyer, M.D.; Altenbach, R.J.; Basha, F.Z.; Carroll, W.A.; Condon, S.; Elmore, S.W.; Kerwin, J.F.; Sippy, K.B.; Tietje, K.; Wendt, M.D.; et al. Structure-activity studies for a novel series of tricyclic substituted hexahydrobenz $[e]$ isoindole $\alpha 1 \mathrm{~A}$ adrenoceptor antagonists as potential agents for the symptomatic treatment of benign prostatic hyperplasia (BPH). J. Med. Chem. 2000, 43, 1586-1603.

7. Cardile, A.P.V.; Santagati, A.; Gentile, B. Thienopyrimidine derivatives prevent cartilage destruction in articular disease. Farmaco 2001, 56, 959-964.

8. Lee, K.; Sotzing, G.A. Poly(thieno[3,4- $b]$ thiophene). A new stable low band gap conducting polymer. Macromolecules 2001, 34, 5746-5747.

9. Heeney, M.; Bailey, C.; Genevicius, K.; Shkunov, M.; Sparrowe, D.; Tierney, S.; McCulloch, U. Stable polythiophene semiconductors incorporating thieno[2,3-b]thiophene. J. Am. Chem. Soc. 2005, 127, 1078-1079.

10. Elguero, J. Comprehensive Heterocyclic Chemistry; Katritzky, A., Ed.; Pergamon Press: Oxford, UK, 1984; Volume 5, p. 277.

11. Elguero, J. Comprehensive Heterocyclic Chemistry; Shintai, I., Ed.; Elsevier: Oxford, UK, 1986; Volume 5, p. 3.

12. Butnariu, R.M.; Caprosu, M.D.; Bejan, V.; Angalagiu, I.I.; Ungureanu, M.; Poiata, A.; Tuchilus, C.; Florescu, M. Pyridazine and phthalazine derivatives with potential antimicrobial activity. J. Heterocycl. Chem. 2007, 44, 1149-1152.

13. Caprosu, M.; Butnariu, R.; Mangalagiu, I.I. Synthesis and antimicrobial activity of some new pyridazine derivatives. Heterocycles 2005, 65, 1871-1879.

14. Dima, S.; Caprosu, M.; Ungureanu, M.; Grosu, G.; Petrovanu, M. New derivatives of 1-methylphthalazine with antimicrobial and fungistatic action. Ann. Pharm. Fr. 1999, 5, 415-416.

15. Dodge, D. Herbicides and Plant Metabolism; Cambridge University Press: Cambridge, UK, 1989; p. 114.

16. Drochioiu, G.; Sunel, V.; Oniscu, C.; Basu, C.; Murariu, M. The breakdown of plant biostructure followed by amino acids determination. Roum. Biotechnol. Lett. 2001, 6, 155-165. 
17. Drochioiu, G.; Strajeriu, S.; Petrovanu, M.; Druta, I. Plant genetic resources newsletter. 2002, $129,47-51$.

18. Druta, I.; Cuciac, C.; Danac, R.; Avram, E.; Rotaru, A.; Drochioiu, G. The phytotoxic effect of some new monoquaternary salts of 4,4'-bipyridyl and 1,10-phenanthroline. Pak. J. Appl. Sci. 2002, 2, 145-150.

19. Druta, I.; Danac, R.; Ungureanu, M.; Drochioiu, G. Antimicrobial activity of new derivatives of 1,10-phenanthroline, Ann. Pharm. Fr. 2002, 60, 348-351.

20. Gokçe, M.; Dogruer, D.; Sahin, M.F. Synthesis and antinociceptive activity of 6-substituted-3pyridazinone derivatives. Farmaco 2001, 56, 233-237.

21. Mabkhoot, Y.N. Synthesis and analysis of some bis-heterocyclic compounds containing sulphur. Molecules 2009, 14, 1904-1914.

22. Mabkhoot, Y.N.; Kheder, N.A.; Al-Majid, A.M. Facile and convenient synthesis of new thieno[2,3-b] thiophene derivatives. Molecules 2010, 15, 9418-9426.

23. Kheder, N.A.; Mabkhoot, Y.N.; Farag, A.M. Synthesis and antimicrobial evaluation of some bis(thioxopyridine), bis(pyrazolo[3,4- $b]$ pyridine), bis(thieno[2,3-b]pyridine), bis(1,3,4-thiadiazole) and bis(thiophene) derivatives. Heterocycles 2008, 75, 2937-2948.

24. Kheder, N.A. Convenient synthesis of novel bis(hydrazone) and bis(indole) derivatives. Heterocycles 2009, 78, 1281-1288.

25. Kheder, N.A. Synthesis of some novel bis(pyrazole), bis(pyridine) and bis pyrazolo[5,1-c]-1,2,4triazine derivatives. Heterocycles 2009, 78, 1815-1822.

26. Wolkoff, P. A new method of preparing hydrazonyl halides. Can. J. Chem. 1975, 53, 1333-1335.

27. Dieckmann, W.; Platz, L. By a new methode of formation of Osotetrazonen. Chem. Ber. 1905, 38, 2986-2990.

28. Eweiss, N.F.; Osman, A. Synthesis of heterocycles. Part II. New routes to acetyl thiadiazolines and alkylazothiazoles. J. Heterocycl. Chem. 1980, 17, 1713-1717.

29. Shawali, A.S.; Eweiss, N.F.; Hassaneen, H.M.; Al-gharib, M.S. Synthesis and rearrangement of ethyl aryloxyglyoxalate arylhydrazones. Bull. Chem. Soc. Jpn. 1975, 48, 365-366.

Sample Availability: Samples of the compounds 5-11 are available from the authors.

(C) 2013 by the authors; licensee MDPI, Basel, Switzerland. This article is an open access article distributed under the terms and conditions of the Creative Commons Attribution license (http://creativecommons.org/licenses/by/3.0/). 